\title{
Designs of Langmuir probes for W7-X
}

\author{
Ralph Laube ${ }^{\mathrm{a}}$, Michael Laux ${ }^{\mathrm{a}}$, Min You Ye ${ }^{\mathrm{a}}$, Henri Greuner ${ }^{\mathrm{b}}$, Stefan Lindig ${ }^{\mathrm{b}}$ \\ ${ }^{a}$ Max-Planck-Institut für Plasmaphysik, EURATOM Association, Teilinstitut Greifswald, \\ Wendelsteinstr. 1, D-17491 Greifswald, Germany \\ ${ }^{b}$ Max-Planck-Institut für Plasmaphysik, EURATOM Association, \\ Boltzmannstr. 2, D-85748 Garching, Germany
}

\begin{abstract}
Several designs of Langmuir probes for the stellarator Wendelstein 7-X (W7-X) are described. Different types of probes are proposed for the different divertors to be used during different operational phases of W7-X. Comblike arrays of stiff probes, arrays of flexible probes, and fixed inlay probes are reviewed. For the initial phase of $\mathrm{W} 7-\mathrm{X}$ it was decided to install arrays of fixed inlay probes. Two mockups were manufactured and one of them was tested with success in the high heat flux test facility GLADIS. For long-pulse operation of W7-X different conceptual designs are proposed and are still developed further. This paper summarizes the different design constrains for the Langmuir probes in the different divertor surroundings, describes the design of the array of inlay probes for the initial phase and the result of the GLADIS test, and gives a preview of the conceptual designs of probes for the long-pulse operational phase of $\mathrm{W} 7-\mathrm{X}$.
\end{abstract}

Keywords: Langmuir probe, Stiff probe array, Flexible probe array, Fixed inlay probe, Popup probe, Stellarator

\section{Introduction}

Observing the density, the electron temperature, and the floating potential of the divertor plasma of W7-X Langmuir probes are an essential tool for the diagnostics. A Langmuir probe consists of a conducting tip exposed to the plasma and insulated otherwise that is biased against the vacuum vessel via a power supply. This allows to apply different voltages to the tip and to measure the resulting currents. The desired plasma parameters can be deduced from the so obtained voltagecurrent characteristic [1].

During the initial phase of W7-X an inertially cooled test divertor unit (TDU) [2] will be installed, whereas for the later operation with long pulses an actively cooled high heat flux divertor (HHF) is foreseen [3].

For W7-X, with its fivefold symmetry and similar divertors in each module of the machine, it is proposed to install Langmuir probes in the two horizontal divertor blades of module 5. To monitor the strike line of the plasma impact a distribution of several probes (constituting a probe array) crossing the strike zone seems appropriate. As in a stellarator the strike zones can vary depending on the operational conditions two arrays of Langmuir probes have been planned for each horizontal divertor blade: one between target modules 2 and 3 (or within target 3 during the TDU operation), the other one between modules 7 and 8 (omitted for the TDU) [Fig. 1].

As a first attempt a cylindrical pneumatic actuator was applied to move a carrier over a distance of at least $6 \mathrm{~mm}$ and pull it back within $0.2 \mathrm{~s}$. Such a pneumatic system was designed [4] and a prototype was manufactured and tested. But reasonable doubts about the applicability of the pneumatic system (air pressure and moving parts in ultra high vacuum, necessity of current carrying movable connectors in the presence of ECRH radiation and magnetic field, no possibility to compensate the displacements of the divertor caused by thermal expansion) caused a review of the design concept for the probes resulting in the development of a more appropriate new solution for the TDU.

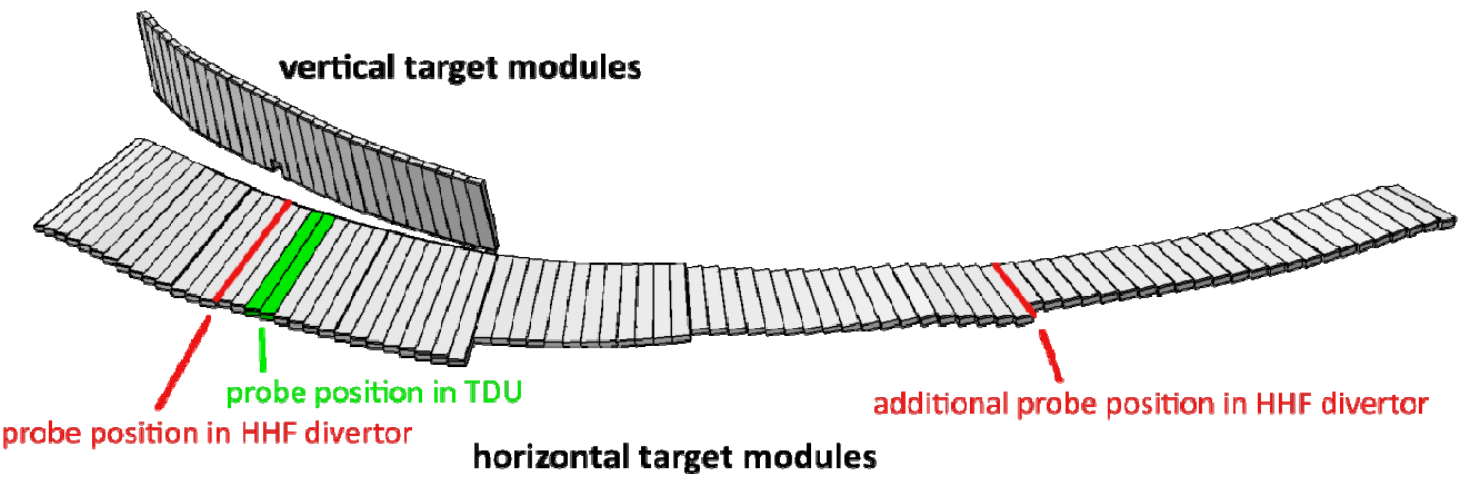

Fig. 1 Positions of the Langmuir probe arrays in the TDU and HHF divertors 


\section{Design requirements}

The technical specification for in-vessel components, the restrictions for materials used in W7-X, and the requirements of the probe diagnostics define the general design constraints:

- operation under vacuum $\left(10^{-8} \mathrm{mbar}\right)$

- pump down to $<5 \times 10^{-6}$ mbar within $<48 \mathrm{~h}$

- magnetic fields $\leq 3 \mathrm{~T}$

- ECRH (microwave) radiation

- poloidal distance between adjacent tips $\sim 15 \mathrm{~mm}$

- well defined collecting area of the tip

- well defined positions w.r.t. the target surface

- proximity to the strike zone (profile)

- electromagnetic shielding of the probes

- relative permeability $\mu_{\mathrm{r}}<1.01$

- average cobalt content $<500$ ppm

Following the functional specification of in-vessel components for the initial phase of W7-X, additional constraints for the Langmuir probe design are due to the following points:

- no (easy) service access to the divertor rear

- un-cooled divertor tiles consisting of graphite (thickness $\sim 30 \mathrm{~mm}$ )

- max. power density at divertor strike line $8 \mathrm{MW} / \mathrm{m}^{2}$

- max. surface temperature of graphite tiles: $1800^{\circ} \mathrm{C}$ (front), $600^{\circ} \mathrm{C}$ (back side)

- vertical displacements of the target surface up to $1.5 \mathrm{~mm}$ possible

The additional constraints for the HHF divertor can be gathered from the technical specification for the target modules of W7-X divertor:

- divertor tiles consisting of a $19 \mathrm{~mm}$ thick heat sink out of $\mathrm{CuCrZr}$ covered by a CFC layer (thickness $8-10 \mathrm{~mm}$ ) via a $3 \mathrm{~mm}$ bonding $\mathrm{Cu}$ interlayer

- max. power density at steady state mode $10 \mathrm{MW} / \mathrm{m}^{2}$ for $30 \mathrm{~min}$.

- max. power density $15 \mathrm{MW} / \mathrm{m}^{2}$ for $5 \mathrm{~s}$

\section{Design of Langmuir probes for the TDU}

\subsection{Conceptual designs}

There are two principle ways to design the Langmuir probes - as a moving or a static system. The static system consists of the probe body mounted into other structures but electrically insulated and a wiring to the outside. By contrast, for a moving system an actuator is indispensable (inside or outside the vessel). Magnetic drives (exploiting $\mathrm{I} \times \mathrm{B}$ forces) are restricted to currents small enough not to distort the local magnetic configuration in the plasma. Piezoelectric drives seemed to be a favorable alternative to the pneumatic actuators, but they are still not available for temperatures above $150{ }^{\circ} \mathrm{C}$. Furthermore, there has to be a positioning system that allows adopting an exact position of the probe tips with respect to the vessel. Of course a sophisticated probe tip mounted on an individual spring and equipped with an end stop can do the job. But in conclusion of the comparison of the concepts the static solution seems much simpler than a flexible probe array.

For the static concept fixed inlay probe tips (FIP) were introduced directly into a divertor target, which means that the probe bodies have to be insulated from the imbedding itself. To confirm the feasibility of the FIPs a rough model was designed for a finite element analysis of the thermo-mechanical behavior. As a prerequisite all thermal and mechanical properties (and their dependences on temperature) have to be provided. The thermal contact resistance between graphite and the selected insulator (AlN plates, $0.65 \mathrm{~mm}$ thick) had to be determined experimentally as a function of contact pressure and temperature. The FE calculation based on a simplified CAD model and using the standard case operational parameters confirmed the feasibility of the FIP solution [Fig. 2] [5].

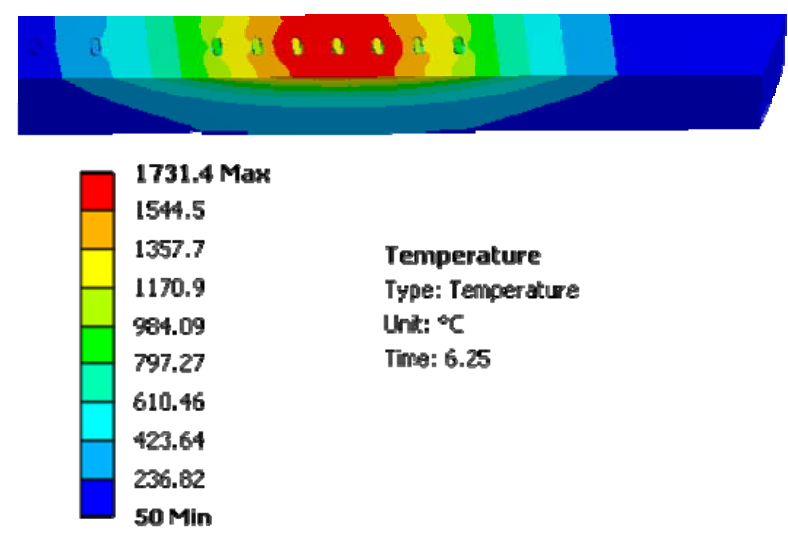

Fig. 2 FE simulation of a target with fixed inlay probes

\subsection{Design of the FIP-solution}

\subsubsection{The target integrated tips}

The FIP-array consists of a standard TDU target, which is milled out, in order to insert probe bodies at distinct positions along the target. These targets have basic dimensions of $570 \times 55 \times 30 \mathrm{~mm}(\mathrm{~L} \times \mathrm{W} \times \mathrm{H})$. The surface structure of the target determines the tip distance of $25 \mathrm{~mm}$. To reach the demanded resolution of $15 \mathrm{~mm}$ two adjacent targets are equipped with probes. Because of a $10 \mathrm{~mm}$ shift of surface structures between these two targets the resulting resolution of the interspaced tips meets the demanded one. The targets selected for instrumentation are nos. 3 and 4 of the 3rd target module of the horizontal divertor blade. Each target is provided with ten probes, five of them to both sides of a middle bar belonging to a support structure behind the target module. Thus there are ten intarsia along a length of $160 \mathrm{~mm}$ on each side of the middle bar, interrupted by a $60 \mathrm{~mm}$ distance given by the width of the bar [Fig. 3].

To avoid internal mechanical strains due to differing thermal expansion the probe tips are made of the same material as the surrounding target. As for intarsia the plasma facing surface of the tip fits into the target surface. It has an oval geometry of $12 \mathrm{~mm}$ length and $3 \mathrm{~mm}$ width. 


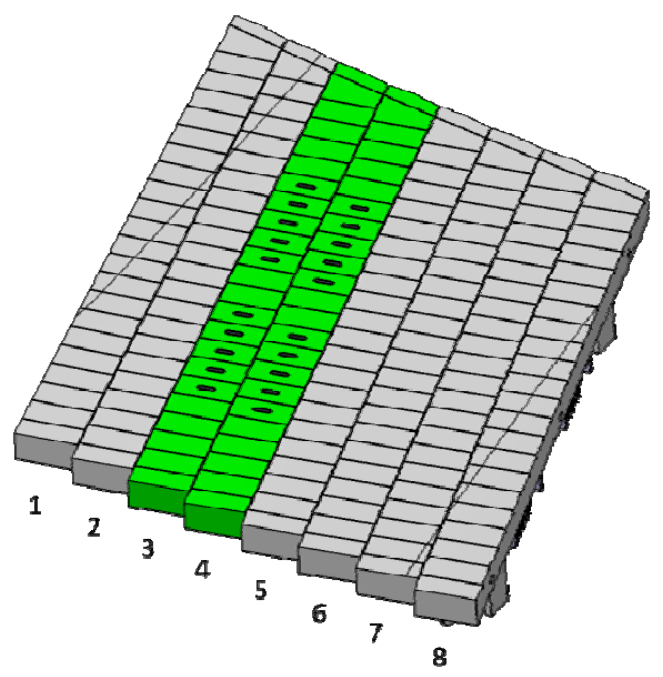

Fig. 3 Probes in target module 3 (horizontal TDU blade)

The tip is isolated from the imbedding oval hole by a small $(\approx 0.7 \mathrm{~mm})$ gap filled with isolating plates made of aluminum nitride of $0.65 \mathrm{~mm}$ thickness. These insulator plates do not reach the plasma facing edge to avoid carbon deposition onto it that could result in an electric short. Aluminum nitride is a ceramic isolator with a heat conductivity as high as $180 \mathrm{~W} / \mathrm{m} \cdot \mathrm{K}$, which makes it possible to dissipate the incident power to the target back efficiently without creating an electric short.

Still the instrumentation is expected to weaken the thermo-mechanical properties of the target, therefore, target material was chosen for the instrumented target that with slightly improved parameters as compared to the standard target graphite. Its heat conductivity amounts to $130 \mathrm{~W} / \mathrm{m} \cdot \mathrm{K}$ and the thermal expansion coefficient is $4.1 \times 10^{-6} \mathrm{~K}^{-1}$ (standard graphite has a heat conductivity of $110 \mathrm{~W} / \mathrm{m} \cdot \mathrm{K}$ and a thermal expansion coefficient of $5.2 \times 10^{-6} \mathrm{~K}^{-1}$ ). Disk springs made from Inconel are used to hold the probes in position during operation.

The layout of the instrumented target containing the Langmuir probe bodies is shown in [Fig. 4]

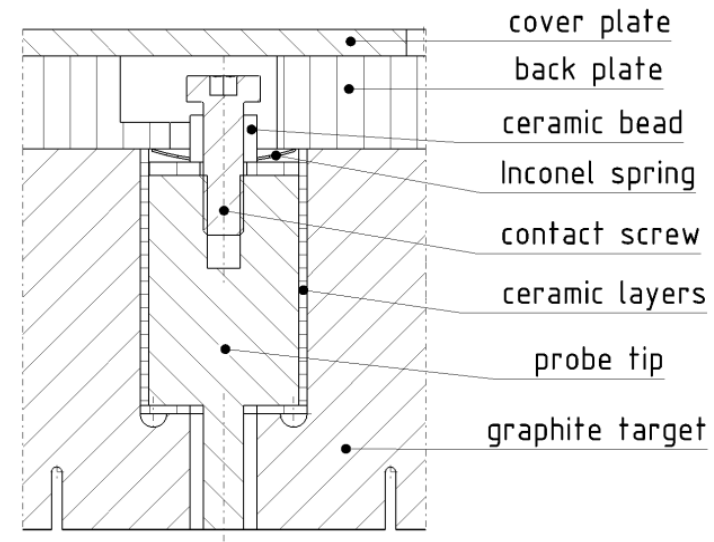

Fig. 4 Cross section of a probe tip in the TDU target

The plasma facing surfaces of all divertor targets (and, consequently, also the probes) are cut by a free form surface to follow the magnetic surface of the plasma as close as possible. This means that in principle each target and each probe tip are individual items. To simplify manufacturing the plasma facing surface of instrumented targets should be milled with the probe tips installed. Then the raw bodies of all tips can be made in a batch production, which reduces efforts and costs.

\subsubsection{Design of the cabling on the target module}

On the back side of the target each probe tip is connected to the wiring (cable with a diameter of $0.8 \mathrm{~mm}$ ) by contact screws. Screwing was favored over soldering because of the high temperatures expected for the back side of the un-cooled divertor. Those temperatures also exclude a conventional insulation of the cables, like Kapton. For this reason ceramic beads are threaded onto the wires. To avoid cross-talking the cables of the respective probes must be screened against each other. To realize this, a system of tunnels is milled into the backside of the non-magnetic stainless steel back-plates. They accommodate the ceramic-insulated conductors and screen them against each other. After mounting the tunnels are closed by a steel cover-plate. A bundle of $8 \times 1$ copper tubes is used to guide the cables from these back-plates to the edge of the target module reaching a plug-connector unit.

\subsubsection{Design of the connector-unit on the module}

It is planned that the arrays are installed together with the entire target module embodying the arrays. This unit is assembled outside of the plasma vessel and then introduced and mounted as a whole. This assembly procedure forces the introduction of an electrical interface at the end of the target module. This interface is designed as a pair of plug-connectors collecting the cables from the whole array to simplify the assembly. 50-pin D-Sub connectors are used, consisting of a ceramic body to withstand high temperatures. For the same reason crimp shells are used instead of soldering.

\subsubsection{Design of the in-vessel cabling}

Starting from the connections at the end of the target module the cables of the probes are routed (screened against each other) along the vessel wall into the port and until the port flange in a dedicated tube. For each probe a screened double-core cable is used (max. diameter $1.8 \mathrm{~mm}$ ). These are routed in two $18 \times 1$ copper tubes between divertor and in-vessel wall and - from there on - in a $22 \times 1 \mathrm{Cu}$-tube. Owing to manufacturing tolerances as well as displacements caused by differing thermal expansions of different parts of the tubing, flexible non-magnetic stainless steel hoses will be interposed between tube sections. In order to protect the cable against ECRH radiation and, simultaneously, to ensure an acceptable evacuation of the entire tubes, holes with $0.5 \mathrm{~mm}$ diameter are drilled into the mantle of the tubes by a laser ( 4 holes on the circumference for every $10 \mathrm{~mm}$ tube length).

\subsubsection{Design port and feed trough}

At the port flange (DN100 CF) all probe cables are connected to the outside via two 50-pin D-Sub feed trough-connectors integrated into the flange. As for 
other connections in the vessel the vacuum requirements have to be met and compatibility with the impact of the ECRH stray radiation has to be assured. Additionally, the screening of the individual probe cables against each other has to be maintained. To keep the area necessary for assembly as small as possible no additional cap is introduced on top of the port. Instead a ring is welded into the port tube, to separate the last part of the tube. The incoming cables are threaded through the central hole of a circular disc. The end of the guiding tube consisting of a flexible hose is screwed into this hole. After the mounting of the two D-Sub connectors the disc is plunged into the port-tube and screwed to the ring. The emerging separated port volume containing the cable ends and the connectors is now tight against ECRH radiation. A sufficient number of evacuation holes have to be laser drilled into the disc.

\subsection{GLADIS test}

In order to confirm the design of the FIPs, several tests of a first mockup of the probe array have been carried out in the GLADIS facility [6]. During the tests the expected power input of $8 \mathrm{MW} / \mathrm{m}^{2}$ for $6.25 \mathrm{~s}$ was simulated by a hydrogen ion beam. The surface of the target was heated up to approximately $1700{ }^{\circ} \mathrm{C}$ [Fig. 5]. A possible overloading was simulated with $13 \mathrm{MW} / \mathrm{m}^{2}$ whereby a still acceptable surface temperature of approximately $2100^{\circ} \mathrm{C}$ was reached. The tests demonstrated that the design of the FIPs can take the loads expected for the initial operating phase of W7-X.

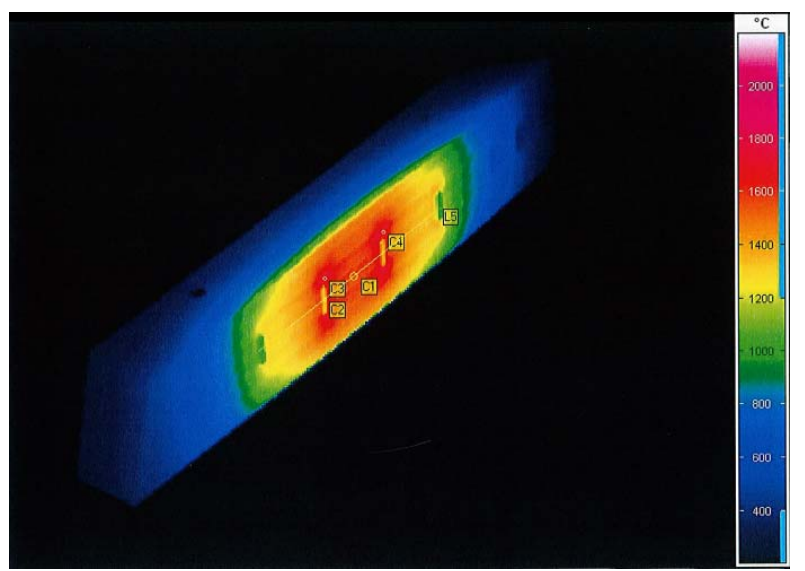

Fig. 5 Infrared camera view during GLADIS test, $8 \mathrm{MW} / \mathrm{m}^{2}$, $6.25 \mathrm{~s}$, start temperature $200{ }^{\circ} \mathrm{C}$

For an ultimate test the probe bodies were removed from the target and the remaining skeleton was loaded with a power of $10 \mathrm{MW} / \mathrm{m}^{2}$. Even then the target neither did exhibit any incorrect characteristics nor was it eroded pointing to the fact that the thermal conduction along the target is not as important as suspected. Consequently, the inclined sides of the probe bodies (truncated pyramids) could be replaced by a cuboid to simplify the probe geometry. A second mock-up with the simplified and cost saving new structure will follow shortly.

\section{Conceptual designs of Langmuir probes for the HHF Divertor}

Unlike for the TDU, the probes tips for the HHF divertor cannot be installed easily into the target elements directly because they possibly would interfere with the embedded cooling structures. Accordingly, movable probe arrays are proposed as an appropriate approach to a probe diagnostics for the long-pulse operational phase of W7-X. The only way to position the probes into the divertor is to install the arrays within the narrow gaps between adjacent target modules. Such arrays are designed for the gaps between modules 2 and 3 as well as modules 7 and 8, respectively.

A substantial advantage for the operation of probes in the HHF divertor is that the targets surrounding the probes are cooled. Therefore, it is sufficient to remove the probe tips after exposure with a small stroke of approximately $10 \mathrm{~mm}$ from a zone of high heat load into an actively cooled area. Furthermore, it is expected that the back side of the actively cooled divertor will stay well below $100{ }^{\circ} \mathrm{C}$. Therefore, any actuator unit has to withstand only the temperatures reached during the baking of the plasma vessel (about $150{ }^{\circ} \mathrm{C}$ ). This allows for piezoelectric drives as an alternative to the pneumatic solutions since actuators with Curie temperatures around $320^{\circ} \mathrm{C}$ (which can resist a permanent temperature of up to $150{ }^{\circ} \mathrm{C}$ ) are readily available. Because motions of a single piezo-actuator are very short fast piezo-motor solutions are needed to drive the probe arrays.

Apart from pneumatics and piezo-actuators drives based on the Lorentz force acting on a current carrying conductor in a crossing magnetic field are analyzed for their possibilities and restrictions. In that case the magnetic field of the stellarator is used to produce a force on a revolvable loop carrying a switched current to move a probe tip to its measuring position and back. A stop position at the back of the target secures the accurate measuring position of the probe tip with respect to the back side of the target.

\section{Conclusion}

The design phase of the Langmuir probes at the W7-X for the initial TDU phase is nearly finished. A first mock-up was successfully tested; a second mock-up is still pending. For the long-pulse operating phase of W7-X the design of the Langmuir probes did not start yet, but a review of different conceptual solutions for movable probes especially for the drive principles (pneumatic, piezo, Lorentz-force) is well under way.

\section{References}

[1] N. Hershkowitz, How Langmuir Probes Work, in: O. Auciello, D. L. Flamm (eds), Plasma Diagnostics Discharge Parameters and Chemistry 1, Academic Press, Boston, MA. (1989) 113- 83

[2] A. Peacock, et al., Fusion Engineering and Design 84, 1475-1478 (2009)

[3] H. Greuner, et al., Fusion Engineering and Design 84, 848852 (2009)

[4] A. Reichert, Diploma Thesis FH Stralsund, 2002

[5] M.Y. Ye, et al., Contrib. Plasma Phys. 50, No. 9, 1 - 6 (2010)

[6] H. Greuner, et al., J. Nucl. Mat. 367-370, 1444-1448 (2007) 